N. Toda

Nagoya Math. J.

Vol. 83 (1981), 5-14

\title{
SUR L'INÉGALITÉ FONDAMENTALE DE H. CARTAN POUR LES SYSTÈMES DE FONCTIONS ENTIÈRES
}

\author{
NOBUSHIGE TODA
}

\section{§1. Introduction}

Soit $f=\left(f_{0}, f_{1}, \cdots, f_{n}\right)(n \geqq 1)$ un système transcendant dans le plan $|z|<\infty$; c'est-à-dire, les fonctions $f_{0}, f_{1}, \cdots, f_{n}$ sont entières sans zéros communs à toutes et

$$
\lim _{r \rightarrow \infty} \frac{T(r, f)}{\log r}=\infty
$$

où $T(r, f)$ est la fonction caractéristique de $f$ définie par Cartan ([1]):

$$
T(r, f)=\frac{1}{2 \pi} \int_{0}^{2 \pi} \max _{0 \leq j \leq n} \log \left|f_{j}\left(r e^{i \theta}\right)\right| d \theta-\max _{0 \leq j \leq n} \log \left|f_{j}(0)\right| .
$$

Soient $X$ un ensemble de combinaisons $(\not \equiv 0)$ linéaires, homogènes à coefficients constants de $f_{0}, f_{1}, \cdots, f_{n}$ et linéairement distinctes $n+1$ à $n$ +1 et $\lambda$ le nombre maximum de relations linéaires, homogènes à coefficients constants et linéairement distinctes qui existent entre les $f_{0}, f_{1}, \cdots$, $f_{n}$. On note que $0 \leqq \lambda \leqq n-1$.

Il y a longtemps que Cartan ([1]) a démontré l'inégalité fondamentale suivante quand $\lambda=0$ :

Théorème de Cartan. Pour $q$ combinaisons $F_{1}, \cdots, F_{q}(q \geqq n+2)$ quelconque dans $X$,

$$
(q-n-1) T(r, f)<\sum_{i=1}^{q} N_{n}\left(r, 0, F_{i}\right)+S(r)
$$

où $N_{n}\left(r, 0, F_{i}\right)$ désigne la fonction $N_{n}\left(r, F_{i}\right)$ dans [1] et

$$
S(r)=O(\log r)+O(\log T(r, f)) \quad(r \notin E),
$$

$E$ étant un ensemble de $r$ de mesure linéaire finie.

Et puis il a espéré démontrer l'inégalité suivante quand $\lambda>0$ (originairement pour les fonctions algébroïdes ([1])):

Received June 30, 1978. 
Conjecture de Cartan. Pour $q$ combinaisons $F_{1}, \cdots, F_{q}(q \geqq n+\lambda$ +2 ) quelconque dans $X$,

$$
(q-n-\lambda-1) T(r, f)<\sum_{i=1}^{q} N_{n-\lambda}\left(r, 0, F_{i}\right)+S(r) .
$$

On ne sait pas si cette conjecture serait vraie à présent.

Il y a quelques années, C. Sung ([3]) a donné une proposition plus précise que cette conjecture, mais malheureusement, on peut donner un contre exemple pour cette proposition facilement et elle n'est pas vraie.

Pour cette conjecture, on a donné quelques résultats. Par exemple,

$$
\text { I. } \quad(q-n-1-\lambda(n-\lambda)) T(r, f)<\sum_{i=1}^{q} N_{n-\lambda}\left(r, 0, F_{i}\right)+S(r) .
$$

II. S’il y a $\lambda+1$ combinaisons étant proportionnelles dans $X$,

$$
(q-n-\lambda-1) T(r, f)<\sum_{i=1}^{q} N\left(r, 0, F_{i}\right)+S(r) .
$$

(Dans ce théorème, on peut échanger $N\left(r, 0, F_{i}\right)$ pour $N_{n-\lambda}\left(r, 0, F_{i}\right)$ facilement.)

Dans ce mémoire, on démontre que la conjecture de Cartan est vraie quand $q=n+\lambda+2$ sans autre restriction. De plus, on donne quelques résultats concernant les combinaisons exceptionnelles au sens de Nevanlinna.

On utilise les symboles usuels de la théorie de Nevanlinna ([2]).

\section{§2. Préliminaires}

1. Soient $f, X$ et $\lambda$ comme dans l'introduction. D'abord on note qu'il est facile à démontrer que le nombre maximum de relations linéaires, homogènes à coefficients constants et linéairement distinctes entre $n+1$ combinaisons quelconque dans $X$ est aussi $\lambda$. Soient $F_{1}, \cdots, F_{q}(q \geqq n+1)$ $q$ combinaisons quelconque dans $X$, alors il y a $n+1-\lambda$ combinaisons linéairement indépendantes sur $C$ dans $\left\{F_{i}\right\}_{i=1}^{q}$ (soient $g_{1}, \cdots, g_{n+1-\lambda}$ telles combinaisons) telles que tous les éléments de $X$ sont représentés par $g_{1}, \cdots, g_{n+1-\lambda}$ à coefficients constants:

$$
F=\alpha_{F 1} g_{1}+\cdots+\alpha_{F n+1-\lambda} g_{n+1-\lambda}
$$

$\left(F \in X, \alpha_{F i} \in C\right)$.

Evidemment, $g=\left(g_{1}, \cdots, g_{n+1-\lambda}\right)$ est un système transcendant dans $|z|$ $<\infty$. On dit que telles $g_{1}, \cdots, g_{n+1-\lambda}$ forment une base de $X$. 
On donne quelques lemmes qui seront utilisés après.

LEMME 1. $|T(r, f)-T(r, g)|<O(1)$.

C'est trivial d'après les définitions de $T(r, f), T(r, g)$ et $g$.

LEMME 2. Soient $H_{1}, \cdots, H_{k}(2 \leqq k \leqq n+1) k$ combinaisons quelconque dans X. Alors,

$$
m\left(r,\left\|H_{1}, \cdots, H_{k}\right\| / H_{1} \cdots H_{k}\right)=S(r),
$$

où $\left\|H_{1}, \cdots, H_{k}\right\|$ signifie le wronskian de $H_{1}, \cdots, H_{k}$. (Voir [1])

2. On peut supposer que $g_{i}=F_{i}(i=1, \cdots, n+1-\lambda)$ sans restriction de généralité. Soit

$$
X^{0}=\left\{F_{i}\right\}_{i=n+2-\lambda}^{q}
$$

et par la relation equivalente " $"$ introduite dans [6], on classifie $X^{0}$.

Soient

$$
\begin{gathered}
X^{0} / \sim=\left\{X_{1}^{0}, \cdots, X_{p}^{0}\right\} \quad(1 \leqq p \leqq n+1-\lambda) \\
A_{t}=\left\{F_{i}(1 \leqq i \leqq n+1-\lambda) ; \text { il y a au moins une } F\right. \text { dans } \\
\left.X_{t}^{0} \text { telle que } \alpha_{F i} \neq 0\right\} \quad(t=1, \cdots, p),
\end{gathered}
$$

où $\alpha_{F i}$ est le coefficient de $F_{i}$ quand on représente $F$ par $F_{1}, \cdots, F_{n+1-\lambda}$ (voir (1));

$$
\begin{gathered}
A_{0}=\left\{F_{i}\right\}_{i=1}^{n+1-2}-\bigcup_{t=1}^{p} A_{t}, \\
\nu_{t}=\text { le nombre d'éléments dans } A_{t}(t=0,1, \cdots, p) .
\end{gathered}
$$

On sait que

i) $A_{t_{1}} \cap A_{t_{2}}=\phi \quad\left(t_{1} \neq t_{2}\right)$,

ii) $\sum_{t=0}^{p} \nu_{t}=n+1-\lambda$.

LEMME 3. Quand $q \geqq n+\lambda+2, p=1$ et $\nu_{0}=0$.

Démonstration. Il suffit de prouver ce lemme dans le cas $q=n+\lambda$ +2 parce que $p$ et $\nu_{0}$ diminuent quand $q$ augmente par définition de la relation " $\sim$ ". Supposons qu'il existent au moins deux classes n'étant pas vides entre $A_{0}, A_{1}, \cdots, A_{p}$. Soient $X_{1}^{0}=\left\{F_{n+1-2+j}\right\}_{j=1}^{\mu}$ et $A_{1}=\left\{F_{1}, \cdots, F_{v_{1}}\right\}$ sans restriction de généralité. Alors,

$$
\nu_{1} \leqq n-\lambda \text { et } \mu \leqq \lambda .
$$


NOBUSHIGE TODA

En effet, maintenant, $\nu_{0} \neq 0$ ou $\nu_{2} \neq 0$ et par ii), on a $\nu_{1} \leqq n-\lambda$. Et puis, si $\mu>\lambda$, le nombre maximum de relations linéaires, homogènes à coefficients constants et linéairement distinctes entre les $F_{1}, \cdots, F_{\nu_{1}}$, $F_{n+2-\lambda}, \cdots, F_{n+2}$ est au moins $\lambda+1$ parce que, pour chaque élément dans $X_{1}^{0}$, quand on répresente par $F_{1}, \cdots, F_{n+1-\lambda}$, tous les coefficients de $F_{\nu_{1}+1}$, $\cdots, F_{n+1-\lambda}$ sont égaux à nul. En considérant que $\nu_{1}+\lambda+1 \leqq n+1$, c'est contraire à la définition de $\lambda$. Par conséquent, il faut que $\mu \leqq \lambda$.

D'autre part, pour chaque élément dans $X^{0}-X_{1}^{0}$, tous les coefficients de $F_{1}, \cdots, F_{\nu_{1}}$ sont egaux à nul quand on représente par $F_{1}, \cdots, F_{n+1-\lambda}$ et le nombre des éléments dans $X^{0}-X_{1}^{0}$ est $2 \lambda+1-\mu$ qui est plus grand que ou égal à $\lambda+1$ parce que $\mu \leqq \lambda$. Cela veut dire qu'il existent au moins $\lambda+1$ relations linéaires, homogènes à coefficients constants et linéairement distinctes entre les $F_{\nu_{1}+1}, \cdots, F_{n+1-\lambda}, F_{n+1-\lambda+\mu+1}, \cdots, F_{n+2+\mu}$. En considérant que $n+1-\lambda-\nu_{1}+\lambda+1=n+2-\nu_{1} \leqq n+1$, c'est aussi contraire à la définition de $\lambda$.

Cela veut dire qu'il faut $p=1$ et $\nu_{0}=0$.

\section{§3. Théorèmes}

Soient $f, X$ et $\lambda$ comme dans l'introduction.

THÉORÈmE 1. Soient $F_{1}, \cdots, F_{n+2+2} n+\lambda+2$ combinaisons quelconque dans $X$, alors on a

$$
T(r, f)<\sum_{i=1}^{n+\lambda+2} N_{n-\lambda}\left(r, 0, F_{i}\right)+S(r)
$$

Démonstration. On peut supposer que $F_{1}, \cdots, F_{n+1-\lambda}$ forment une base de $X$. On représente $F_{j}(j=n+2-\lambda, \cdots, n+\lambda+2) \operatorname{par} F_{1}, \cdots, F_{n+1-\lambda}$. Pour simplifier, on met $F_{n+1-\lambda+k} \equiv H_{k}$.

$$
H_{k}=\alpha_{k 1} F_{1}+\cdots+\alpha_{k n+1-\lambda} F_{n+1-\lambda} \quad(k=1, \cdots, 2 \lambda+1) .
$$

I. D'après le lemme 3 , il y a au moins une combinaisons dans $\left\{H_{1}, \cdots\right.$, $\left.H_{2 \lambda+1}\right\}$ telle que le nombre de coefficients différents de zéro à (2) est au moins deux. On peut supposer que $H_{1}$ est telle combinaison.

Soient

$$
\begin{gathered}
\alpha_{1 i(1)_{1}} \neq 0, \cdots, \alpha_{1 i(1)_{m_{1}}} \neq 0 \\
\alpha_{1 i}=0\left(i \neq i(1)_{1}, \cdots, i(1)_{m_{1}}\right) \quad\left(2 \leqq m_{1} \leqq n+1-\lambda\right) .
\end{gathered}
$$

C’est-à-dire, 


$$
H_{1}=\alpha_{1 i(1)} F_{i(1))_{1}}+\cdots+\alpha_{1 i(1)_{1}} F_{i(1) m_{1}} .
$$

Alors, de (3), on obtient

$$
\alpha_{1 i(1) m} F_{i(1) m}=H_{1} \Delta_{1}^{m} / \Delta_{1} \quad\left(m=1, \cdots, m_{1}\right)
$$

où

$$
\Delta_{1}=\left\|F_{i(1)_{1}}, \cdots, F_{i(1)_{m_{1}}}\right\| / F_{i(1)_{1}} \cdots F_{i(1)_{m_{1}}}
$$

et

$$
\Delta_{1}^{m}=\frac{\left\|F_{i(1)_{1}}, \cdots, F_{i(1)_{m-1}}, H_{1}, F_{i(1)_{m+1}}, \cdots, F_{i(1)_{1} m_{1}}\right\|}{F_{i(1)_{1}} \cdots F_{i(1)_{m-1}} H_{1} F_{i(1)_{m+1}} \cdots F_{i(1)_{m_{1}}}} .
$$

De (4), on a l'inégalité

$$
\max _{\alpha_{1 i \neq 0}} \log \left|F_{i}\right| \leqq \log \left|H_{1}\right|+\log ^{+}\left|\frac{1}{\Delta_{1}}\right|+\sum_{m=1}^{m_{1}} \log ^{+}\left|\Delta_{1}^{m}\right|+O(1) .
$$

Quand $m_{1}=n+1-\lambda$, en intégrant à régard de $\theta$ de 0 jusqu'à $2 \pi$, on a l'inégalité suivante d'après les lemmes 1 et 2 comme d'habitude:

$$
\begin{aligned}
T(r, f) & <N\left(r, 0, H_{1}\right)+m\left(r, 1 / \Delta_{1}\right)+\sum_{m=1}^{m_{1}} m\left(r, \Delta_{1}^{m}\right)+O(1) \\
= & N\left(r, 0, H_{1}\right)+\sum_{m=1}^{m_{1}} N\left(r, 0, F_{i(1)_{m}}\right)-N\left(r, 0,\left\|F_{i(1)_{1}}, \cdots, F_{i(i)_{1} m_{1}}\right\|\right)+S(r) \\
\leqq & N_{n-2}\left(r, 0, H_{1}\right)+\sum_{i=1}^{n+1-2} N_{n-2}\left(r, 0, F_{i}\right)+S(r) .
\end{aligned}
$$

II. Quand $m_{1}<n+1-\lambda$, d'après le lemme 3, il y a une combinaison $H_{k}$ dans $\left\{H_{2}, \cdots, H_{2 \lambda+1}\right\}$ et un $i$ tels que

$$
H_{1} \approx H_{k}, \quad \alpha_{1 i}=0 \text {, et } \alpha_{k i} \neq 0,
$$

où la relation " $\approx$ " est définie dans [6]. On peut supposer que $H_{k}=H_{2}$.

Soient

$$
\begin{gathered}
\left\{i ; \alpha_{1 i}=0, \alpha_{2 i} \neq 0\right\}=\left\{i(2)_{1}, \cdots, i(2)_{m_{2}}\right\}, \\
\Delta_{2}=\left\|H_{2}, F_{i(2) 1}, \cdots, F_{i(2)_{m_{2}}}\right\| / H_{2} F_{i(2)_{1}} \cdots F_{i(2)_{m_{2}}}
\end{gathered}
$$

et

$$
\Delta_{2}^{m}=\frac{\left\|H_{2}, F_{i(2)_{1}}, \cdots, F_{i(2)_{m-1}}, \tilde{F}_{2}, F_{t(2)_{m+1}}, \cdots, F_{i(2)_{m}}\right\|}{H_{2} F_{i(2)_{1}} \cdots F_{i(2)_{m-1}} \tilde{F}_{2} F_{i(2)_{m+1}} \cdots F_{i(2)_{m}}}
$$

où 


$$
\tilde{F}_{2} \equiv-\sum_{\substack{\alpha i \neq 0 \\ \alpha_{2 i} \neq 0}} \alpha_{2 i} F_{i}=-H_{2}+\sum_{\substack{\alpha i=0 \\ \alpha 2 i \neq 0}} \alpha_{2 i} F_{i}
$$

On note que

$$
\left\{i ; \alpha_{1 i} \neq 0, \alpha_{2 i} \neq 0\right\} \neq \phi \quad \text { et } \quad \tilde{F}_{2} \neq 0
$$

parce que $H_{1} \approx H_{2}$. Alors, on obtient

$$
\alpha_{2 i(2)_{m}} F_{i(2)_{m}}=\tilde{F}_{2} \Delta_{2}^{m} / \Delta_{2} \quad\left(m=1, \cdots, m_{2}\right) .
$$

De (7), on a l'inégalité

$$
\max _{\substack{\alpha_{1 i}=0 \\ \alpha_{2 i} \neq 0}} \log \left|F_{i}\right| \leqq \log \left|\tilde{F}_{2}\right|+\log ^{+}\left|\frac{1}{\Delta_{2}}\right|+\sum_{m=1}^{m_{2}} \log ^{+}\left|\Delta_{2}^{m}\right|+O(1)
$$

et de (6)

$$
\log \left|\tilde{F}_{2}\right| \leqq \max _{\substack{\alpha_{1 i} \neq 0 \\ \alpha_{2 i} \neq 0}} \log \left|F_{i}\right|+O(1) \leqq \max _{\alpha_{1 i} \neq 0} \log \left|F_{i}\right|+O(1) .
$$

Quand $m_{1}+m_{2}=n+1-\lambda$, de (5), (8) et (9), on a

$$
\begin{aligned}
\max _{1 \leqq i \leqq n+1-\lambda} \log \left|F_{i}\right| \leqq & \log \left|H_{1}\right|+\log ^{+}\left|\frac{1}{\Delta_{1}}\right|+\log ^{+}\left|\frac{1}{\Delta_{2}}\right|+\sum_{m=1}^{m_{1}} \log ^{+}\left|\Delta_{1}^{m}\right| \\
& +\sum_{m=1}^{m_{2}} \log ^{+}\left|\Delta_{2}^{m}\right|+O(1),
\end{aligned}
$$

de sorte qu'en intégrant à régard de $\theta$ de 0 jusqu'à $2 \pi$ on obtient l'inégalité suivante d'après les lemmes 1 et 2 :

$$
\begin{aligned}
T(r, f)< & N\left(r, 0, H_{1}\right)+m\left(r, 1 / \Delta_{1}\right)+m\left(r, 1 / \Delta_{2}\right) \\
& +\sum_{m=1}^{m_{1}} m\left(r, \Delta_{1}^{m}\right)+\sum_{m=1}^{m_{2}} m\left(r, \Delta_{2}^{m}\right)+O(1) \\
= & N\left(r, 0, H_{1}\right)+N\left(r, 0, H_{2}\right)+\sum_{i=1}^{n+1-\lambda} N\left(r, 0, F_{i}\right) \\
& -N\left(r, 0,\left\|F_{i(1)_{1}}, \cdots, F_{i(1)_{1}}\right\|\right)-N\left(r, 0,\left\|H_{2}, F_{i(2)_{1}}, \cdots, F_{i(2)_{m}}\right\|\right)+S(r)
\end{aligned}
$$

et en tenant compte de la multiplicité des zéros de $H_{1}, H_{2}, F_{1}, \cdots, F_{n+1-\lambda}$

$$
\leqq N_{n-\lambda}\left(r, 0, H_{1}\right)+N_{n-\lambda}\left(r, 0, H_{2}\right)+\sum_{i=1}^{n+1-\lambda} N_{n-\lambda}\left(r, 0, F_{i}\right)+S(r)
$$

III. Quand $m_{1}+m_{2}<n+1-\lambda$, d'après le lemme 3, il y a au moins une $H_{j}$ dans $\left\{H_{3}, \cdots, H_{2 \lambda+1}\right\}$ et un $i$ tels que

$$
H_{1} \approx H_{j} \text { ou } H_{2} \approx H_{j}
$$

et 


$$
\alpha_{1 i}=0, \quad \alpha_{2 i}=0, \quad \alpha_{j i} \neq 0 .
$$

On peut supposer que $H_{j}=H_{3}$. Soient

$$
\begin{aligned}
& \left\{i ; \alpha_{i 1}=\alpha_{2 i}=0, \alpha_{3 i} \neq 0\right\}=\left\{i(3)_{1}, \cdots, i(3)_{m_{3}}\right\}, \\
& \Delta_{3}=\left\|H_{3}, F_{i(3)_{1}}, \cdots, F_{i(3)_{m}}\right\| / H_{3} F_{i(3)_{1}} \cdots F_{i(3)_{3}}, \\
& \Delta_{3}^{m}=\frac{\left\|H_{3}, F_{i(3)_{1}}, \cdots, F_{i(3)_{m-1}}, \tilde{F}_{3}, F_{i(3)_{m+1}}, \cdots, F_{i(3)_{m}}\right\|}{H_{3} F_{i(3)_{1}} \cdots F_{i(3)_{m-1}} \tilde{F}_{3} F_{i(3)_{m+1}} \cdots F_{i(3)_{m}}}
\end{aligned}
$$

où

$$
\tilde{F}_{3} \equiv-\sum_{\substack{\alpha_{3 i} \neq 0 \\ \alpha_{1 i} \neq 0 \text { ou } \alpha_{2 i} \neq 0}} \alpha_{3 i} F_{i}=-H_{3}+\sum_{\substack{\alpha_{3 i} \neq 0 \\ \alpha_{1 i}=\alpha_{2 i}=0}} \alpha_{3 i} F_{i} .
$$

Parce que $H_{1} \approx H_{2}$ ou $H_{2} \approx H_{3}$,

$$
\left\{i ; \alpha_{3 i} \neq 0,\left|\alpha_{1 i}\right|+\left|\alpha_{2 i}\right| \neq 0\right\} \neq \phi \quad \text { et } \quad \tilde{F}_{3} \not \equiv 0 \text {. }
$$

Alors, on a

$$
\alpha_{3 i(3) m} F_{i(3) m}=\tilde{F}_{3} \Delta_{3}^{m} / \Delta_{3} \quad\left(m=1, \cdots, m_{3}\right)
$$

et de cela, on a

$$
\max _{\substack{\alpha_{3 i} \neq 0 \\ \alpha_{1 i}=\alpha_{2 i}=0}} \log \left|F_{i}\right| \leqq \log \left|\tilde{F}_{3}\right|+\log ^{+}\left|\frac{1}{\Delta_{3}}\right|+\sum_{m=1}^{m_{3}} \log ^{+}\left|\Delta_{3}^{m}\right|+O(1)
$$

et

$$
\log \left|\tilde{F}_{3}\right| \leqq \max _{\alpha_{1 i} \neq 0 \text { ou } \alpha_{2 i} \neq 0} \log \left|F_{i}\right|+O(1) .
$$

Des inégalités (5), (8), (9), (10) et (11), on a

$$
\max _{\substack{\alpha 1 i \neq 0 \text { ou } \alpha_{3 i} \neq 0 \\ \text { ou } \alpha_{3 i} \neq 0}} \log \left|F_{i}\right| \leqq \log \left|H_{1}\right|+\sum_{k=1}^{3} \log ^{+}\left|\frac{1}{\Delta_{k}}\right|+\sum_{k=1}^{3} \sum_{m=1}^{m_{k}} \log ^{+}\left|\Delta_{k}^{m}\right|+O(1) .
$$

Quand $m_{1}+m_{2}+m_{3}=n+1-\lambda$, en considérant que

$$
\left\{i ; \alpha_{1 i} \neq 0 \text { ou } \alpha_{2 i} \neq 0 \text { ou } \alpha_{3 i} \neq 0\right\}=\{1,2, \cdots, n+1-\lambda\},
$$

on a l'inégalité suivante comme dans II:

$$
T(r, f)<\sum_{k=1}^{3} N_{n-\lambda}\left(r, 0, H_{k}\right)+\sum_{i=1}^{n+1-\lambda} N_{n-\lambda}\left(r, 0, F_{i}\right)+S(r) .
$$

IV. En general, en tenant compte du lemme 3, il existe un nombre $s(1 \leqq s \leqq 2 \lambda+1)$ tel que

$$
m_{1}+m_{2}+\cdots+m_{s}=n+1-\lambda,
$$


où $m_{1}, m_{2}, \cdots, m_{s}$ sont définis comme dans $\mathrm{I} \sim \mathrm{III}$. Alors,

$$
\left\{F_{i} ; \alpha_{1 i} \neq 0 \text { ou } \alpha_{2 i} \neq 0 \text { ou } \cdots \text { ou } \alpha_{s i} \neq 0\right\}=\left\{F_{i}\right\}_{i=1}^{n+1-2},
$$

par conséquent, comme dans III, on a

$$
\max _{1 \leqq i \leqq n+1-2} \log \left|F_{i}\right| \leqq \log \left|H_{1}\right|+\sum_{k=1}^{s} \log ^{+}\left|\frac{1}{\Delta_{k}}\right|+\sum_{k=1}^{s} \sum_{m=1}^{m_{k}} \log ^{+}\left|\Delta_{k}^{m}\right|+O(1)
$$

et comme dans II,

$$
T(r, f)<\sum_{k=1}^{s} N_{n-\lambda}\left(r, 0, H_{k}\right)+\sum_{i=1}^{n+1-\lambda} N_{n-\lambda}\left(r, 0, F_{i}\right)+S(r) .
$$

Quand $s<2 \lambda+1$, en ajoutant

$$
\sum_{k=s+1}^{2 \lambda+1} N_{n-\lambda}\left(r, 0, H_{k}\right) \quad(\geqq 0)
$$

à la droite à (12), on a

$$
T(r, f)<\sum_{i=1}^{n+2+2} N_{n-2}\left(r, 0, F_{i}\right)+S(r)
$$

parce que $H_{k}=F_{n+1-\lambda+k}(k=1, \cdots, 2 \lambda+1)$.

N.B. 1. En général, on ne peut pas échanger le nombre " $n+\lambda+2$ " pour un autre nombre plus petit. (Voir l'exemple dans [6].)

Pour $F$ dans $X$, on met

$$
\delta(F)=1-\limsup _{r \rightarrow \infty} \frac{N(r, 0, F)}{T(r, f)} .
$$

Quand $\delta(F)>0$, on dit que $F$ est exceptionnelle au sens de Nevanlinna.

Corollaire. Pour $F_{1}, \cdots, F_{n+\lambda+2} n+\lambda+2$ combinaisons quelconque dans $X$,

$$
\sum_{i=1}^{n+\lambda+2} \delta\left(F_{i}\right) \leqq n+\lambda+1
$$

([6], Corollaire 2).

Ici, on considère le cas où l'égalité est réalisée dans ce corollaire.

LEMME 4. S'il existent $F_{1}, \cdots, F_{n+1+\mu}$ dans $X$ telles que

$$
\sum_{i=1}^{n+1} \delta\left(F_{i}\right)+\delta\left(F_{n+1+j}\right)>n+1 \quad(j=1, \cdots, \mu)
$$

alors, $\lambda \geqq \mu$.

([6], Lemme 8) 
THÉORÈme 2. Quand $\lambda>0$, s'il existent $\left\{F_{i}\right\}_{i=1}^{n+\lambda+2}$ dans $X$ telles que

$$
\sum_{i=1}^{n+\lambda+2} \delta\left(F_{i}\right)=n+\lambda+1,
$$

alors, il existe un $i_{0}$ tel que

$$
\delta\left(F_{i_{0}}\right)=0 \quad \text { et } \quad \delta\left(F_{i}\right)=1 \quad\left(i \neq i_{0}\right) .
$$

Démonstration. On peut supposer sans restriction de généralité:

$$
\delta\left(F_{1}\right) \geqq \delta\left(F_{2}\right) \geqq \cdots \geqq \delta\left(F_{n+\lambda+2}\right) .
$$

$\mathrm{Si}$

$$
\delta\left(F_{n+\lambda+2}\right)>0
$$

alors,

$$
\delta\left(F_{n+\lambda+1}\right)<1,
$$

Par conséquent, de (13), on a les inégalités suivantes:

$$
\sum_{i=1}^{n+1} \delta\left(F_{i}\right)+\delta\left(F_{n+1+1}\right)>n+1 \quad(j=1, \cdots, \lambda+1) .
$$

Alors, du Lemme 4,

$$
\lambda \geqq \lambda+1,
$$

qui est absurde. Cela veut dire qu'il faut

$$
\delta\left(F_{n+2+2}\right)=0
$$

et de (13),

$$
\delta\left(F_{i}\right)=1 \quad(i=1, \cdots, n+\lambda+1) .
$$

N.B. 2. Dans ce cas, l'ordre de $f$ est positif entièr ou infini et $f$ est à croissance régulière ([4], Théorème 3).

\section{BIBLIOGRAPHIE}

[1] H. Cartan, Sur les zéros des combinaisons linéaires de $p$ fonctions holomorphes données, Mathematica 7 (1933), 5-31.

[2] R. Nevanlinna, Le théorème de Picard-Borel et la théorie des fonctions méromorphes, Gauthier-Villars, Paris 1929.

[ 3 ] C. Sung, Contributions to holomorphic curves in complex manifolds, Thèse 1975.

[4] N. Toda, Sur la croissance de fonctions algébroïdes à valeurs déficientes, Kōdai Math. Sem. Rep. 22 (1970), 324-337. 
[5] N. Toda, Sur quelques combinaisons linéaires exceptionnelles au sens de Nevanlinna, Tôhoku Math. J., 23 (1971), 67-95.

[6] - Sur quelques combinaison linéaires exceptionnelles au sens de Nevanlinna, V, Nagoya Math. J., 66 (1977), 37-52.

Université de Nagoya 